her first examination, nothing unusual was noted about her, except that she was "rather dull and heavy". The disorder was general, but not very violent. She had had it for three months; it was her second attack, the former one having taken place two years prior. She had never menstruated; the pulse was low; the bowels had been confined for some days; they were always torpid; the jactitations were said to continue during sleep, which was subsequently proved to be the fact.

In accordance with my usual plan of treatment, she was duly purged with our compound magnesia draught, had a turpentine enema, and then some form of iron.

On the 25 th, she became much more violent in her muscular contortions, and talked wildly, though perfectly sensible to questions and making rational answers. This state, with the addition of pervigilance, continued with little variation for nearly a fortnight, during which time came gradually on those typhus-like appearances noted in the other cases; the prostration being, however, far greater in this one. So incessant were the involuntary muscular movements, that at the bend of the elbows the cuticle got chafed and abraded, and from this extended a bright superficial erysipelatous redness as high as the armpits. Nothing controlled for a moment the violence of the symptoms; night and day she writhed and jerked and twisted about. By November 5th, it was quite evident that she would sink from exhaustion, for up to that time she took stimulants and nourishment more easily than I was at all prepared for or can readily explain, since the muscles of the throat were affected with the rest. The low state of her vitality was furtber evidenced now by the appearance of a large slough on the sacrum, and a gangrenous hue on the abrasions at the elbows; and so her powers of endurance gradually wore out, and she died on the 12th. There was no stupor, nor other sign of cerebral effusion, until a few hours before her death.

Remarks. It is apparent to me from the issue of this case, that both of the other ones I have related were much nearer to a similar ending than $I$ at all supposed; and $I$ am still at a loss to see how they escaped from it. As a matter of fact, until within a week or two of the end, this fatal case did not wear so threatening an aspect in some particulars as either of the other two, especially the first I alluded to.

The pathology of this case, and of all similar ones, is a point of great interest; for what are the structural changes, if any, or the morbid appearances, discoverable in the cerebro-spinal axis from such a disorder? I cannot say what they were in the case before us, as a post mortem examination was peremptorily refused. I do not imagine, however, that we should have found anything more than serous effusion into the arach. noid, and possibly into the ventricles of the brain; and even that most probably merely as the result of disordered vascular action a few hours prior to death. We shall have no right, I think, to assign to any particular portion of the nervous centres any particular lesion or structural change as the exciting cause of the symptoms; whilst seeing what the symp. toms were from first to last, we shall have still less right to expect to find any of the products of pure inflammatory action. The important practical fact which I deduce from the case is, that simple brain-irritation, prolonged, it may be, to the spinal marrow, apart from any inflammatory action either in themselves or their meninges, may induce some of the same phy. sical results, and that exhaustion consequent upon long-continued nervous excitement and spasmodic muscular actions may kill a patient with St. Vitus' dance, just as it does in hy. drophobia, or some of the forms of tetanus.

\section{Briatinal Communication.}

\section{AN OMALOUS CASE OF TYPHOID FEVER.}

By T. OGIER WARD, M.D., Winkfield, near Windsor.

ON August 7th, I was requested to see a delicate female child, about 2 years old, one of a family of six children, four of which, boys, had died from teething at about the same age. I was told she had had a bad cold for a week, attended with cough and violent running from the eyes and nose, and slight sorethroat. She had lost her appetite, was feverish, and disinclined to quit the arms of her mother or the nurse. She slept badly, with slight occasional delirium; but this was only evinced by her striking her mother on one or two occasions when disturbed at night. Her bowels were rather confined. I found her shy and fretful, with a quick pulse, a little more heat of skin than in health, only eight teeth fully cut, but no redness or tenderness of the gums nor flushed cheeks. The tongue was rather white at the back, moist and clean at the tip. She had slight thirst. The abdomen was not distended, nor very hot. Profuse discharge of puro-mucus flowed from both nostrils, which, as she tried to breathe through them, her throat being ob. structed by enlarged tonsils, caused her to respire with diffi. culty, but not rapidly. This difficulty was much increased during sleep.

Thinking I had to treat a mere feverish cold, I prescribed a mercurial aperient and a saline pectoral mixture, which was continued for five days. The cough and fever had at the end of this time subsided, though the discharge from the nose was rather worse, and there was a complete disgust to food of every kind. I ordered iron wine, with infusion of orange peel, which, after three days, was discontinued, as her mother thought it did her no good, and confined her bowels. I then, finding that she still took no food, urged the use of wine, which she took in very small quantities, and with great reluctance after the first day; and, thinking it was possible that the discharge from the nose (which had now become decidedly purulent and very offensive, and had caused small pustules outside the nos. trils; might be a cause of the anorexia, I had the nostrils in jected with a solution of the nitrate of zinc, which in two days completely stopped the discharge and dried up the pustules, but without any improvement to the appetite. This was on August 19th, and I advised the resumption of the iron wine. On the 20th, I found the hands and feet swollen and cold, and the child restless, rubbing its head with its hands; the abdomen, however, being quite contracted. I then inquired whether it had passed urine, and learnt that for some days it had only passed it once in twelve hours. I therefore ordered it a stimu. lant saline diuretic, of which it took a portion, but without any effect, and died, rather suddenly, at 5 , A.x., on the $2(0$ th.

As the symptoms described above, viz., the fever, attended with discharge from the nose and sorethroat- the former persisting after the cessation of the fever and being followed by retention or diminution of urine-with swelling of the hands and feet, seemed to resemble the course of scarlatina, I inquired whether any eruption had been observed at first; but this being denied, I thought it might still be a case of scarlatina without eruption; but a post mortem examination cleared up the mystery by shewing that it was a case of typhoid fever. No other organs than the Peyerian glands of the ileum, and the mesentery, were affected; the former being ulcerated, and the latter greatly enlarged.

As to the origin of the complaint, as the child never went off the premises previously to her illness, and there are no houses within forty yards, the only causes I can suspect are that the child would often stand with her nurse near the sink in the scullery, which is often offensive, and that there is a muddy pond on the same side of the road, about sixty yards distant. The occurrence of a purulent discharge from the nostrils is certainly to me a novel feature of typhoid fever ; and, together with the absence of fever after the first fortnight, rendered the case so obscure, that I think it may be fairly placed among the instances of latent typhoid fever.

\section{CASE OF POISONING BY POTATOE-BERRIES.}

By Thomas Morris, Esq., Merford, Gresford.

ON Tuesday, the 7 th of August, about six, P.s., I was summoned to attend Miss M. E. H., aged 14, the messenger stating she had eaten the berries of the potatoe plant, portions of which she showed me.

On arriving at the bedside of my patient, I found her labouring under the effects of a narcotico-acrid poison. She was tossing to and fro in bed; the skin was bedewed with a cold clammy perspiration, and of a rather livid colour; respiration was hurried; the pulse was exceedingly quick and feeble; the teeth for the most part were closed, and she was constantly spitting, through the closed teeth, a viscid frothy phlegm. She was speechless. She would, after repeated asking, put out her tongue, which was covered with a brown, moist fur. She occasionally asked for drink, and begged her mother not to leave her. The eyes for the most part were open, and the pupils were not very much dilated. The expression was anxious. She occasionally slumbered for a few minutes, and again was restless.

From the history of the case, it appeared she had been slightly indisposed the week before; but was better on the \\ $\frac{\bar{\omega}}{\bar{D}}$


Sunday, and in the afternoon went into the garden and partook of the berries. In the evening she complained of pain in the bowels, and the nurse took her a glass of gin and water and rhubarb pills. On Monday there was a good deal of sickness; but none of the crude vegetable appeared in the ejected matter. On Tuesday the sickness continued, and the matter ejected was of a greenish cast (it may have been bile, or assimilated vegetable matter). An emetic of ipecacuanha was now given by her parent, and the crude vegetable ejected which was shown to me by the messenger when I was hastily sent for.

Considering the length of time that had elapsed since the poison had been taken, together with the fact that a considerable quantity of the vegetable matter had been ejected, I felt it my duty to attend to the depressed vital powers; and, with this object in view, I directed her to take strong coffee, and brandy, and arrowroot, and prepared a mixture consisting of carbonate of ammonia and aromatic spirt of ammonia, in mint water, a dessertspoonful to be taken every half hour, in arrow. root. Brandy and arrowroot were given in the intervals, and I administered a turpentine enema occasionally, which would soon come away, bringing some feculent matter, mixed with portions of the seed.

I remained in attendance all night, and thought the pulse in the morning more firm; but as there still remained much doubt about the case, a desire was shown to have Dr. Williams, of Wrexham, called in, which was at once agreed to. I left about half-past six, A.M., for two hours, and returned in time to meet Dr. Williams (in whose absence Mr. Eyton Jones attended).

There were now more decided symptoms of sinking; the pulse was scarcely perceptible at the wrist, and it was deemed necessary to make an impression on the system. With this view, a mustard plaster was applied over the abdomen, which acted. A dose of turpentine in gruel was administered, and the enema was increased in strength; but she gradually sank, and expired about twelve at noon on Wednesday. There was no post mortem examination.

Reßranks. The hurried respiration and bluish cast of the skin, arose, probably, from the air-cells at the base of the lungs being impervious, through an accumulation of viscid secretion. Would small bleedirgs, by diminishing the volume of blood and enabling the heart to act more perfectly, have been of any utility, considering the depressed state of the circulation and nervous energy? Would such practice be safe? We often find small bleeding, when the system is recovering from a state of collapse, to be of great advantage.

\section{ILLUSTRATIONS OF THE USE OF THE OPHTHALMOSCOPE.}

By Wrltuam Martin, F.R.C.S., late Professor of Ophthalmic Surgery in the Calcutta Medical College.

\section{[Concluded from $p .697$.]}

Parasitic Bodies in the Exe.

By means of the ophthalmoscope, we are able occasionally to detect parasites, animal and vegetable; and these are by no means confined to the anterior parts of the globe. An in teresting case is described and figured in Desmarres (vol. iii, pp. 757.8) of cysticercus in the retina. There were signs of turbid vitreous humour-floating false membranes; the greater portion of the fundus, including the papilla, entirely hid; but, by making the patient look a little below and to the inside, there was discovered a cysticercus thus described. The neck is of a bluish white, evidently agitated at the slightest movement. Once M. Desmarres saw it become shorter, and the head hide itself almost entirely in the body of the tumour. The body is of a brilliant yellow-white colour, seven or eight times the size of the optic papilla.

\section{Injuries of the Eye from Foreign Bodies.}

Mr. White Cooper, in his recently published and beautifully illustrated work on Wounds and Injuries of the Eye, remarks, at page 41 , that the ophthalmoscope, in doubtful cases, may render us valuable assistance. If a chip of metal, for instance, has lodged in the vitreous humour, without traversing or wounding the lens or its capsule, it will readily be discovered by the ophthalmoscope, unless buried in such a position as not to admit of its being brought into view. If the lens has been wounded, it will becone opaque, and of course prevents the use of this apparatus. And he gives extracts from the works of Jaeger, illustrative of this subject; also, at page 233 , he gives instances of the value of this means of observation, in a case in which, after a wound from a cricketball on the globe, sight became extinct; and, on close examination, there were found a number of brownish flakes floating on the vitreous humour; and effusion, varying in parts from pink to a chocolate hue, pervaded the retina, covering the entrance of the optic nerve. A distinct dark clot of blood was discernible near the lower and outer portion of the retina. In another case, by means of this mode of examination, he was enabled to speak of the nature of an injury with confidence and precision, where, without it, he could only have hazarded an opinion of what was probable. The eye of a farmer having been struck by a missile, sight was lost. Externally, nothing abnormal, but a dilated and motionless pupil, was seen. The cphthalmoscope showed the seat of a rent in the retina, as an opaque irregular line, vearly in the axis of vision; and there were many small spots around this, evidently the remains of the coagulum of blood (detected previously by Mr. Dixon) which had not been entirely absorbed. "In our report, we were enabled to state with confidence that, though Mr. J. might retain a certain amount of sight in the injured organ, we were of opinion that it would never be restored to its former perfection."

Among many interesting examples Mr. W. Cooper's book contains of the value of this instrument in the elucidation of injuries of the eyes and their effects, are the following:At page $1 \pm 0$ is related a case, in the practice of Gräfe, of a student in whom, after recovery from injury, the ophthalmoscope showed detachment of the retina in the lower half. The upper half was normal, and the lens and vitreous humour remained transparent. The patient only lost the sight in the upper half of the visual field. At page 163, he gives an in stance in his own practice, in which he was enabled to detect by this means the changes which had taken place in the posterior part of the eye, from an injury, causing retinal effusions etc. He also relates a case at page 176 , where he was enabled to trace double vision, after a blow, to an opaque line on the posterior surface of the capsule; the media otherwiso transparent. At page 178, he gives an instance of a man rendered almost totally amaurotic in consequence of a blow near the eyebrow. In former days, this would have been attributed simply to injury of the frontal nerve and its branches; but Mr. Cooper was enabled to detect partial detachment of the retina, bloody coagula, and other changes much more likely than any injury of the external nerves to cause the amaurosis; in fact, such organic alterations as must be to a great extent incompatible with the due performance of the functions of the retina. At page 180, he gives a case of Mr. Bowman's, in which there were detected, after concussion of the brain, abnormal appearances of the retina and choroid, with numerous black spots both before and behind the retina; another case, under Dr. Williams, in which he found the "retina elevated by a turbid liquid, such as is often observed in pericarditis and other serous inflammations". At page 181 , he gives a case, under the care of Dr. Van Dom mellen, in which, after concussion of the brain, the ophthalmo scope showed the papilla replaced by a reddish disc; veins alternately raised and depressed; arteries very small and pale. At page 235 , in a case in which, after a blow, the patient saw a dark cloud before his sight, Mr. Cooper detected a greenish grey mass at the posterior part of the vitreous humour, obscuring the retina. As the eye moved, this was jerked up, and then settled slowly down-evidently a coagulum of blood, intercepting the rays of light.

In concluding these notes, we may remark, that formerly it was one of the most rare things to have an opportunity of verifying by subsequent or post mortem examination any morbid changes which had been supposed to have taken place in the eye, with the exception of cases of malignant tumours for which the globe had been extirpated. Now that it is the practice of ophthalmic surgeons to remove diseased globes more often than formerly, instead of leaving them in the orbit, to create irritation and endanger the existence of the sound eye and that more pains are taken to form collections of the diseased globes which are removed,- the facilities of comparing diseased appearances with the revelations of the ophthalmoscope have become vastly increased, and thus a wide field is opened for improvement of our knowledge of this branch of science, and particularly in the minute anatomy and pathology of the regions; and, as a result, for more decisive and more certain methods of practice.

24, George Street, Hanover Square, August 1859. 